\title{
Naturalness versus stringy naturalness with implications for collider and dark matter searches
}

\author{
Howard Baer $\odot,{ }^{1, *}$ Vernon Barger, ${ }^{2, \dagger}$ and Shadman Salam ${ }^{1, *}$ \\ ${ }^{1}$ Homer L. Dodge Department of Physics and Astronomy, University of Oklahoma, Norman, Oklahoma 73019, USA \\ ${ }^{2}$ Department of Physics, University of Wisconsin, Madison, Wisconsin 53706, USA
}

(Received 21 June 2019; published 3 September 2019)

\begin{abstract}
The notion of stringy naturalness - that an observable $\mathcal{O}_{2}$ is more natural than $\mathcal{O}_{1}$ if more (phenomenologically acceptable) vacua solutions lead to $\mathcal{O}_{2}$ rather than $\mathcal{O}_{1}$-is examined within the context of the Standard model (SM) and various supersymmetric models (SUSY) extensions: Constrained minimal supersymmetric standard model (CMSSM)/minimal supergravity model (mSUGRA), high-scale SUSY, and radiatively driven natural SUSY (RNS). Rather general arguments from string theory suggest a (possibly mild) statistical draw towards vacua with large soft SUSY breaking terms. These vacua must be tempered by an anthropic veto of nonstandard vacua or vacua with too large a value of the weak scale $m_{\text {weak }}$. We argue that the SM, the CMSSM and various high-scale SUSY models are all expected to be relatively rare occurrences within the string theory landscape of vacua. In contrast, models with $\mathrm{TeV}$-scale soft terms but with $m_{\text {weak }} \sim 100 \mathrm{GeV}$ and consequent light higgsinos (SUSY with radiatively driven naturalness) should be much more common on the landscape. These latter models have a statistical preference for $m_{h} \simeq 125 \mathrm{GeV}$ and strongly interacting sparticles beyond current LHC reach. Thus, while conventional naturalness favors sparticles close to the weak scale, stringy naturalness favors sparticles so heavy that electroweak symmetry is barely broken and one is living dangerously close to vacua with charge-or-color breaking minima, no electroweak breaking or pocket universe weak-scale values too far from our measured value. Expectations for how landscape SUSY would manifest itself at collider and dark matter search experiments are then modified compared to usual notions.
\end{abstract}

DOI: 10.1103/PhysRevResearch.1.023001

\section{INTRODUCTION}

Naturalness. The gauge hierarchy problem (GHP) [1]what stabilizes the weak scale so that it does not blow up to the GUT/Planck scale-is one of the central conundrums of particle physics. Indeed, it provides crucial motivation for the premise that new physics should be lurking in or around the weak scale. Supersymmetric models (SUSY) with weak-scale soft SUSY breaking terms provide an elegant solution to the GHP [2,3] but so far weak-scale sparticles have failed to appear at the CERN Large Hadron Collider (LHC) and WIMPs have failed to appear in direct detection experiments [4]. The latest search limits from LHC Run 2 require gluinos with $m_{\tilde{g}} \gtrsim 2.25 \mathrm{TeV}$ [5] and top squarks $m_{\tilde{t}_{1}} \gtrsim 1.1 \mathrm{TeV}$ [6]. These lower bound search limits stand in sharp contrast to early sparticle mass upper bounds from naturalness that seemingly required $m_{\tilde{g}, \tilde{t}_{1}} \lesssim 0.4 \mathrm{TeV}$ [7-10]. ${ }^{1}$ Thus LHC limits seem to imply the soft SUSY breaking scale $m_{\text {soft }}$ lies in the multi-TeV rather than the weak-scale range. This then opens

\footnotetext{
*baer@ou.edu

†barger@pheno.wisc.edu

‡shadman.salam@ou.edu
}

Published by the American Physical Society under the terms of the Creative Commons Attribution 4.0 International license. Further distribution of this work must maintain attribution to the author(s) and the published article's title, journal citation, and DOI.

${ }^{1}$ For a recent analysis in the context of EENZ/BG, see, e.g., Ref. [11]. up a Little hierarchy problem (LHP) [12]: why does the weak scale not blow up to the energy scale associated with soft SUSY breaking, i.e., why is $m_{\text {weak }} \ll m_{\text {soft }}$ ?

Early upper bounds on sparticle masses derived from naturalness were usually computed using the EENZ/BG logarithmic-derivative measure [7,8]: for an observable $\mathcal{O}$, then

$$
\Delta_{\mathrm{BG}}(\mathcal{O}) \equiv \max _{i}\left|\frac{\partial \ln \mathcal{O}}{\partial \ln p_{i}}\right|=\max _{i}\left|\frac{p_{i}}{\mathcal{O}} \frac{\partial \mathcal{O}}{\partial p_{i}}\right|,
$$

where the $p_{i}$ are fundamental parameters of the underlying theory. For an observable depending linearly on model parameters, $\mathcal{O}=a_{1} p_{1}+\cdots+a_{n} p_{n}$, then $\partial \mathcal{O} / \partial p_{i}=a_{i}$ and $\Delta_{\mathrm{BG}}(\mathcal{O})$ just picks off the maximal right-hand-side contribution to $\mathcal{O}$ and compares it to $\mathcal{O}$. In the case where one contribution $a_{i} p_{i} \gg \mathcal{O}$, then some other contribution(s) will have to be finetuned to large opposite-sign values such as to maintain $\mathcal{O}$ at its measured value. Such finetuning of fundamental parameters seems highly implausible in nature absent some symmetry or parameter selection mechanism. Thus the logarithmic derivative is a measure of [13] practical naturalness. An observable $\mathcal{O}$ is natural if all independent contributions to $\mathcal{O}$ are comparable to or less than $\mathcal{O}$.

For the case of the LHP, the observable $\mathcal{O}$ is traditionally take to be $m_{Z}^{2}$ and the $p_{i}$ are taken to be the MSSM $\mu$ term and soft SUSY breaking terms so that naturalness then requires all contributions to $m_{Z}^{2}$ to be comparable to $m_{Z}^{2}$ : this is the basis for expecting sparticles to occur around the $100-\mathrm{GeV}$ scale. A fundamental issue in computing $\Delta_{\mathrm{BG}}$ is [14-17]: what is the correct choice to be made for the $p_{i}$ ? If soft terms 
are correlated with one another, as expected in fundamental supergravity/superstring theories, then one gets a very different answer than if one assumes some effective $4-d$ SUSY theory with many independent soft parameters which are introduced to parametrize one's ignorance of their origin. ${ }^{2}$ Alternatively, even if the parameters $p_{i}$ are independent, it is possible that some selection mechanism is responsible for the values towards which they tend.

It is also common in the literature to apply practical naturalness to the Higgs mass:

$$
m_{h}^{2} \simeq m_{H_{u}}^{2} \text { (weak) }+\mu^{2}(\text { weak })+\text { mixing }+ \text { rad. corr., }
$$

where the mixing and radiative corrections are both comparable to $m_{h}^{2}$. Also, $m_{H_{u}}^{2}($ weak $)=m_{H_{u}}^{2}(\Lambda)+\delta m_{H_{u}}^{2}$ where it is common to estimate $\delta m_{H_{u}}^{2}$ using its renormalization group equation (RGE) by setting several terms in $d m_{H_{u}}^{2} / d t$ (with $t=\ln Q^{2}$ ) to zero so as to integrate in a single step:

$$
\delta m_{H_{u}}^{2} \sim-\frac{3 f_{t}^{2}}{8 \pi^{2}}\left(m_{Q_{3}}^{2}+m_{U_{3}}^{2}+A_{t}^{2}\right) \ln \left(\Lambda^{2} / m_{\mathrm{soft}}^{2}\right) .
$$

Taking $\Lambda \sim m_{\mathrm{GUT}}$ and requiring the high-scale measure

$$
\Delta_{\mathrm{HS}} \equiv \delta m_{H_{u}}^{2} / m_{h}^{2} \text {. }
$$

$\Delta_{\mathrm{HS}} \lesssim 1$ then requires three third generation squarks lighter than $500 \mathrm{GeV}[18,19]$ (now highly excluded by LHC topsquark searches) and small $A_{t}$ terms (whereas $m_{h} \simeq 125 \mathrm{GeV}$ typically requires large mixing and thus multi- $\mathrm{TeV}$ values of $\left.A_{0}[20,21]\right)$. The simplifications made in this calculation ignore the fact that $\delta m_{H_{u}}^{2}$ is highly dependent on $m_{H_{u}}^{2}(\Lambda)$ (which is set to zero in the simplification) $[14,16,17]$. In fact, the larger one makes $m_{H_{u}}^{2}(\Lambda)$, then the larger becomes the canceling correction $\delta m_{H_{u}}^{2}$. Thus these terms are not independent: one cannot tune $m_{H_{u}}^{2}(\Lambda)$ against a large contribution $\delta m_{H_{u}}^{2}$. Thus weak-scale top squarks and small $A_{t}$ are not required by naturalness.

To ameliorate the above naturalness calculational quandaries, a more model independent measure $\Delta_{\mathrm{EW}}$ was introduced $[22,23] .{ }^{3}$ By minimizing the weak-scale SUSY Higgs potential, including radiative corrections, one may relate the measured value of the $Z$-boson mass to the various SUSY contributions:

$$
\begin{aligned}
m_{Z}^{2} / 2 & =\frac{m_{H_{d}}^{2}+\Sigma_{d}^{d}-\left(m_{H_{u}}^{2}+\Sigma_{u}^{u}\right) \tan ^{2} \beta}{\tan ^{2} \beta-1}-\mu^{2} \\
& \simeq-m_{H_{u}}^{2}-\mu^{2}-\Sigma_{u}^{u}\left(\tilde{t}_{1,2}\right) .
\end{aligned}
$$

The measure

$$
\Delta_{\mathrm{EW}}=\mid(\max \text { RHS contribution }) \mid /\left(m_{Z}^{2} / 2\right)
$$

\footnotetext{
${ }^{2}$ For instance, in dilaton-dominated SUSY breaking, one expects $m_{0}^{2}=m_{3 / 2}^{2}$ and $m_{1 / 2}=-A_{0}=\sqrt{3} m_{3 / 2}$. In such a case, it would not make sense to adopt $m_{0}$ and $m_{1 / 2}$ as free, independent parameters.

${ }^{3} \mathrm{~A}$ desirable feature of $\Delta_{\mathrm{EW}}$ is that for a given SUSY spectrum, one obtains exactly the same finetuning measure whether the spectrum is generated from multi- or few parameter theory or at the weak scale (such as pMSSM) or at much higher scales. This model independence is not shared by other measures such as $\Delta_{\mathrm{BG}}$ or $\Delta_{\mathrm{HS}}$.
}

is then low provided all weak-scale contributions to $m_{Z}^{2} / 2$ are comparable to or less than $m_{Z}^{2} / 2$. The $\Sigma_{u}^{u}$ and $\Sigma_{d}^{d}$ contain over 40 radiative corrections which are listed in Appendix of Ref. [23]. The conditions for natural SUSY (for, e.g., $\Delta_{\mathrm{EW}}<$ $30)^{4}$ can then be read off from Eq. (6).

(1) The superpotential $\mu$ parameter has magnitude not too far from the weak scale, $|\mu| \lesssim 300 \mathrm{GeV}$. This implies the existence of light higgsinos $\widetilde{\chi}_{1,2}^{0}$ and $\widetilde{\chi}_{1}^{ \pm}$with $m\left(\widetilde{\chi}_{1,2}^{0}, \widetilde{\chi}_{1}^{ \pm}\right) \sim$ $100-300 \mathrm{GeV}$.

(2) $m_{H_{u}}^{2}$ is radiatively driven from large high-scale values to small negative values at the weak scale (SUSY with radiatively driven naturalness or RNS [22]).

(3) Large cancellations occur in the $\Sigma_{u}^{u}\left(\tilde{t}_{1,2}\right)$ terms for large $A_{t}$ parameters which then allow for $m_{\tilde{t}_{1}} \sim 1-3 \mathrm{TeV}$ for $\Delta_{\text {EW }}<30$. The large $A_{t}$ term also lifts the Higgs mass $m_{h}$ into the vicinity of $125 \mathrm{GeV}$. The gluino contributes to the weak scale at two-loop order so its mass can range up to $m_{\tilde{g}} \lesssim 6 \mathrm{TeV}$ with little cost to naturalness [23-25].

(4) Since first/second generation squarks and sleptons contribute to the weak scale through (mainly canceling) $D$ terms, they can range up to $10-30 \mathrm{TeV}$ at little cost to naturalness (thus helping to alleviate the SUSY flavor and CP problems) [26].

By combining dependent soft terms in $\Delta_{\mathrm{BG}}$ or by combining the dependent terms $m_{H_{u}}^{2}(\Lambda)$ and $\delta m_{H_{u}}^{2}$ in $\Delta_{\mathrm{HS}}$, then these measures roughly reduce to $\Delta_{\mathrm{EW}}$ (aside from the radiative contributions $\left.\Sigma_{u, d}^{u, d}\right)$. Since $\Delta_{\mathrm{EW}}$ is determined by the weakscale SUSY parameters, then different models which give rise to exactly the same sparticle mass spectrum will have the same finetuning value (model independence). Using the naturalness measure $\Delta_{\mathrm{EW}}$, then it has been shown that plenty of SUSY parameter space remains natural even in the face of LHC Run 2 Higgs mass measurements and sparticle mass limits [23].

String theory landscape. Weinberg's anthropic solution to the cosmological constant (CC) [27], along with the emergence of the string theory landscape of vacua $[28,29]$, have presented a challenge to the usual notion of naturalness. In Weinberg's view, in the presence of a vast assortment $\left(\gg 10^{120}\right)$ of pocket universes which are part of an eternally inflating multiverse, it may not be so surprising to find ourselves in one with $\Lambda_{c c} \sim 10^{-120} m_{P}^{4}$ since if it were much larger, then the expansion rate would be so large that galaxies would not be able to condense, and life as we know it would be unable to emerge. This picture was bolstered by the discovery of the string theory discretuum of flux vacua $[28,29]$ where each metastable minimum of the string theory scalar potential would have a different value of $\Lambda_{c c}$, and also different laws of physics and perhaps even different space-time dimensions. The number of metastable minima has been estimated at around $10^{500}$ [31] although far larger numbers have also been considered. In such a scenario, then it is possible that many of the constants of nature may take on environmentally determined values rather than being determined by fundamental underlying principles.

One example of the challenge to naturalness comes in the form of split supersymmetry (SS) [32]. In SS, one retains the

\footnotetext{
${ }^{4}$ The onset of finetuning for $\Delta_{\mathrm{EW}} \gtrsim 30$ is visually displayed in Fig. 1 of Ref. [24].
} 
positive features of gauge coupling unification and a WIMP dark matter candidate while eschewing the motivation of naturalness. Then the expected SUSY particle spectrum of SS can contain weak-scale gauginos and higgsinos (which furnish gauge coupling unification and a WIMP dark matter candidate) while allowing most scalars (except the SM-like Higgs doublet) to gain unnatural mass values of perhaps $\tilde{m} \sim 10^{3}-10^{8} \mathrm{TeV}$. Here, one might expect the weak scale to blow up to the $\tilde{m}$ scale, but the anthropic requirement of $m_{\text {weak }} \sim 100 \mathrm{GeV}$ selects a finely tuned scalar spectrum that would otherwise seem unnatural. In a similar vein, the notion of high-scale SUSY has also been entertained wherein all sparticle masses are large and unnatural, perhaps at the $10^{2}-10^{3}$ TeV scale [33-36].

At first glance, one might expect that the string landscape would make BSM physics nonpredictive since: how are we to determine the metastable vacuum minimum that our universe inhabits out of $\sim 10^{500}$ or more choices? To make progress, Douglas and collaborators [37] have advanced the notion of a statistical program for determining BSM physics. In this case, if we can identify statistical trends for the many landscape vacua solutions, then we might be able to determine probabilistically what sort of pocket universe we are likely to live in.

To this end, Douglas has proposed the notion of stringy naturalness [37]: the value of an observable $\mathcal{O}_{2}$ is more natural than a value $\mathcal{O}_{1}$ if more phenomenologically viable vacua lead to $\mathcal{O}_{2}$ than to $\mathcal{O}_{1}$.

If we apply this definition to the cosmological constant, then phenomenologically viable is interpreted in an anthropic context in that we must veto vacua which would not allow galaxy condensation. Out of the remaining viable vacua, we would expect $\Lambda_{c c}$ to be nearly as large as anthropically possible since there is more volume in $\Lambda_{c c}$ space for larger values. Such reasoning allowed Weinberg to predict the value of $\Lambda_{c c}$ to within a factor of a few of its measured value more than a decade before its value was determined from experiment [27].

Our goal in this paper is to examine Douglas' notion of stringy naturalness and to compare and contrast it to the above conventional notions of naturalness. We will examine what naturalness and what stringy naturalness imply for the SM, the scale of SUSY breaking in SUSY models, and for the magnitude of the weak scale. Our central conclusion from stringy naturalness is: the soft SUSY breaking terms should be as large as possible subject to the constraint that the value of the weak scale in various pocket universes-with the MSSM as the low-energy effective theory-not deviate by more than a factor of a few from its measured value. This is in contrast to the conventional measures which tend to favor smaller soft terms comparable to the weak scale. In fact, stringy naturalness in this form with a relatively mild draw to large soft terms statistically favors a light Higgs mass $m_{h} \sim 125 \mathrm{GeV}$ with as yet no sign of sparticles at LHC.

In Sec. II, we present details of how to implement the notion of stringy naturalness including Douglas' notion of a prior distribution of power-law probability increase in soft term values. This is to be combined with a selection criteria enforcing that the value of the weak scale in various pocket universes not deviate from our measured value by a factor of a few. The latter notion has been presented in some detail by calculations of Agrawal et al. [38]. The landscape selection of the cosmological constant, as emphasized by Douglas et al. [30], operates separately from the soft SUSY breaking term/weak-scale selection criteria.

In Sec. III, we examine first what stringy naturalness implies for the (non-SUSY) standard model (SM). We find the $\mathrm{SM}$ - valid up to a cutoff scale $\Lambda_{\mathrm{SM}}$ - to be highly improbable within the landscape for a cutoff $\Lambda_{\mathrm{SM}} \gg m_{\text {weak }}$. In Sec. IV, we argue that the paradigm CMSSM SUSY model should also be relatively rare on the landscape. In Sec. V, we examine the case of the MSSM on the landscape. In this case, there can be significant swaths of model parameters leading to SUSY with radiatively driven naturalness (RNS). In fact, the statistical draw to large soft terms coupled with a weak scale not too far from its measured value, is exactly what is needed for SUSY with radiatively driven naturalness. In Sec. VI, we present arguments as to why unnatural SUSY models such as split SUSY, high-scale SUSY, spread SUSY and minisplit SUSY are likely to be relatively rare occurrences on the landscape. In Sec. VII, we briefy discuss consequences of stringy naturalness for collider and dark matter searches. A summary and our conclusions are presented in Sec. VIII.

The present work is a continuation of a theme started in Ref. [39] where a qualitative picture of landscape SUSY was presented. Probability distributions for Higgs and sparticles masses were derived from landscape considerations in Ref. [40], while in Ref. [13], predictions for landscape SUSY were compared to LHC simplified model searches along with WIMP direct and indirect detection searches. In Ref. [41], similar methods were applied to determination of the PecceiQuinn scale in SUSY axion models. Some complementary investigations on the likelihood of $N=1$ SUSY emerging from the heterotic landscape have been performed in Ref. [42].

\section{PRIOR DISTRIBUTIONS AND SELECTION CRITERIA FOR LANDSCAPE SUSY}

A simple ansatz for the distribution of string vacua in terms of SUSY breaking scales $m_{\text {hidden }}^{2}$ (where the soft breaking mass scale $m_{\text {soft }}=m_{\text {hidden }}^{2} / m_{P}$ and $m_{P}$ is the reduced Planck mass) is given by

$$
\begin{aligned}
& d N_{\text {vac }}\left[m_{\text {hidden }}^{2}, m_{\text {weak }}, \Lambda_{c c}\right] \\
& \quad=f_{\text {SUSY }}\left(m_{\text {hidden }}^{2}\right) \cdot f_{\text {EWFT }} \cdot f_{c c} \cdot d m_{\text {hidden }}^{2}
\end{aligned}
$$

The cosmological constant finetuning penalty is expected to be $f_{c c} \sim \Lambda_{c c} / m^{4}$ where initial expectations were that $m^{4}$ was taken to be $m_{\text {hidden }}^{4}$. In the $4 d$ supergravity effective theory, which emerges after string compactification, the cosmological constant is given by

$$
\Lambda_{c c}=m_{\text {hidden }}^{4}-3 e^{K / m_{P}^{2}}|W|^{2} / m_{P}^{2},
$$

where $m_{\text {hidden }}^{4}=\sum_{i}\left|F_{i}\right|^{2}+\frac{1}{2} \sum_{\alpha} D_{\alpha}^{2}$ is a mass scale associated with the hidden sector (and usually in SUGRA-mediated models it is assumed $m_{\text {hidden }} \sim 10^{12} \mathrm{GeV}$ such that the gravitino gets a mass $\left.m_{3 / 2} \sim m_{\text {hidden }}^{2} / m_{P}\right)$.

A key observation of Susskind [43] and Denef and Douglas [44] (DD) was that $W$ at the minima is distributed uniformly as a complex variable, and the distribution of $e^{K / m_{P}^{2}}|W|^{2} / m_{P}^{2}$ is not correlated with the distributions of $F_{i}$ and $D_{\alpha}$. Setting the cosmological constant to nearly zero, then, has no effect on 
the distribution of supersymmetry breaking scales. Physically, this can be understood by the fact that the superpotential receives contributions from many sectors of the theory, supersymmetric as well as non-supersymmetric. In this case, the $m^{4}$ in $f_{c c}$ should be taken to be $m_{\text {string }}^{4}$ instead of $m_{\text {hidden }}^{4}$, rendering this term inconsequential to how the number of vacua are distributed in terms of $m_{\text {soft }}$.

Another key observation from examining flux vacua in Sec. II B string theory is that the SUSY breaking $F_{i}$ and $D_{\alpha}$ terms are likely to be uniformly distributed-in the former case as complex numbers, while in the latter case as real numbers. Then one expects the following distribution of supersymmetry breaking scales

$$
f_{\text {SUSY }}\left(m_{\text {hidden }}^{2}\right) \sim\left(m_{\text {hidden }}^{2}\right)^{2 n_{F}+n_{D}-1},
$$

where $n_{F}$ is the number of $F$-breaking fields and $n_{D}$ is the number of $D$-breaking fields in the hidden sector. For just a single $F$-breaking term, then one expects a linear statistical draw towards large soft terms $f_{\text {SUSY }} \sim m_{\text {soft }}^{n}$, where $n=$ $2 n_{F}+n_{D}-1$ and in this case $n=1$. For SUSY breaking contributions from multiple hidden sectors, as typically expected in string theory, then $n$ can be much larger, with a consequent stronger pull towards large soft breaking terms.

An initial guess for $f_{\mathrm{EWFT}}$, the (anthropic) finetuning factor, was $m_{\text {weak }}^{2} / m_{\text {soft }}^{2}$ which would penalize soft terms which were much bigger than the weak scale. This ansatz fails on several points.

(1) Many soft SUSY breaking choices will land one into charge-or-color breaking (CCB) minima of the EW scalar potential. Such vacua would likely not lead to a livable universe and should be vetoed.

(2) Other choices for soft terms may not even lead to EW symmetry breaking (EWSB). For instance, if $m_{H_{u}}^{2}(\Lambda)$ is too large, then it will not be driven negative to trigger spontaneous EWSB. These possibilities also should be vetoed.

(3) In the event of appropriate EWSB minima, then sometimes larger high-scale soft terms lead to more natural weakscale soft terms. For instance, if $m_{H_{u}}^{2}(\Lambda)$ is large enough that EWSB is barely broken, then $\mid m_{H_{u}}^{2}$ (weak) $\mid \sim m_{\text {weak }}^{2}$. Likewise, if the trilinear soft breaking term $A_{t}$ is big enough, then there is large top squark mixing and the $\Sigma_{u}^{u}\left(\tilde{t}_{1,2}\right)$ terms enjoy large cancellations, rendering them $\sim m_{\text {weak }}^{2}$. The same large $A_{t}$ values lift the Higgs mass $m_{h}$ up to the $125-\mathrm{GeV}$ regime.

Here, we will assume a natural solution to the SUSY $\mu$ problem [45]. A recent possibility is the hybrid CCK or SPM models [46], which are based on a $\mathbb{Z}_{24}^{R}$ discrete $R$ symmetry which can emerge from compactification of extra dimensions in string theory. The $\mathbb{Z}_{24}^{R}$ symmetry is strong enough to allow a gravity-safe $U(1)_{P Q}$ symmetry to emerge (which solves the strong CP problem) while also forbidding RPV terms (so that WIMP dark matter is generated). Thus both Peccei-Quinn (PQ) and $R$-parity conservation (RPC) arise as approximate accidental symmetries similar to the way baryon and lepton number conservation emerge accidentally (and likely approximately) due to the SM gauge symmetries. These hybrid models also solve the SUSY $\mu$ problem via a Kim-Nilles [47] operator so that $\mu \sim \lambda_{\mu} f_{a}^{2} / m_{P}$ and $\mu \sim$ 100-200 GeV (natural) for $f_{a} \sim 10^{11} \mathrm{GeV}$ (the sweet zone for axion dark matter). The $\mathbb{Z}_{24}^{R}$ symmetry also suppresses dimension-5 proton decay operators [48].

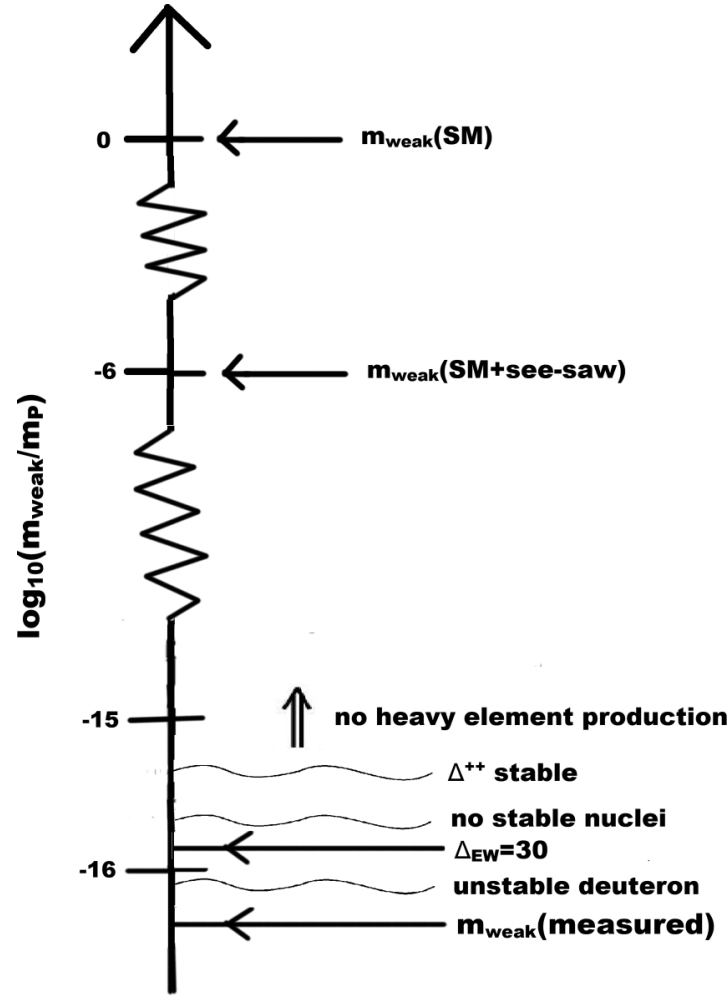

FIG. 1. Value of $m_{\text {weak }}$ as predicted when the SM is valid up to energy scale (1) $Q=m_{P}$, (2) the neutrino see-saw scale, and (3) valid just up to the measured value of $m_{\text {weak }} \sim 100 \mathrm{GeV}$. We also show several anthropic bounds on $m_{\text {weak }}$ from nuclear physics considerations of Agrawal et al. [38].

Once a natural value of $\mu \sim 100-300 \mathrm{GeV}$ is obtained, then we may invert the usual usage of Eq. (6) to determine the value of the weak scale in various pocket universes (with MSSM as low-energy effective theory) for a given choice of soft terms. Based on nuclear physics calculations by Agrawal et al. [38], a pocket universe value of $m_{\text {weak }}^{\mathrm{PU}}$ which deviates from our measured value by a factor $2-5$ is likely to lead to an unlivable universe as we understand it. Weak interactions and fusion processes would be highly suppressed and even complex nuclei could not form. The situation is shown in Fig. 1. We will adopt a conservative value that the weak scale should not deviate by more than a factor four from its measured value. This corresponds to a value of $\Delta_{\text {EW }} \lesssim 30$. Thus, for our final form of $f_{\mathrm{EWFT}}$, we will adopt $f_{\mathrm{EWFT}}=$ $\Theta\left(30-\Delta_{\mathrm{EW}}\right)$ while also vetoing CCB or no EWSB vacua.

\section{WHY THE SM IS LIKELY A RARE OCCURRENCE IN THE LANDSCAPE}

Before using Eq. (7) to evaluate various SUSY models, we will first examine the case of EWSB in the SM. For the SM with

$$
V_{\mathrm{SM}}=-\mu_{\mathrm{SM}}^{2} \phi^{\dagger} \phi+\lambda_{\mathrm{SM}}\left(\phi^{\dagger} \phi\right)^{2},
$$

then the Higgs mass, including quadratic divergent radiative corrections, is found to be

$$
m_{H}^{2} \simeq m_{H}^{2}(\text { tree })+\delta m_{H}^{2},
$$




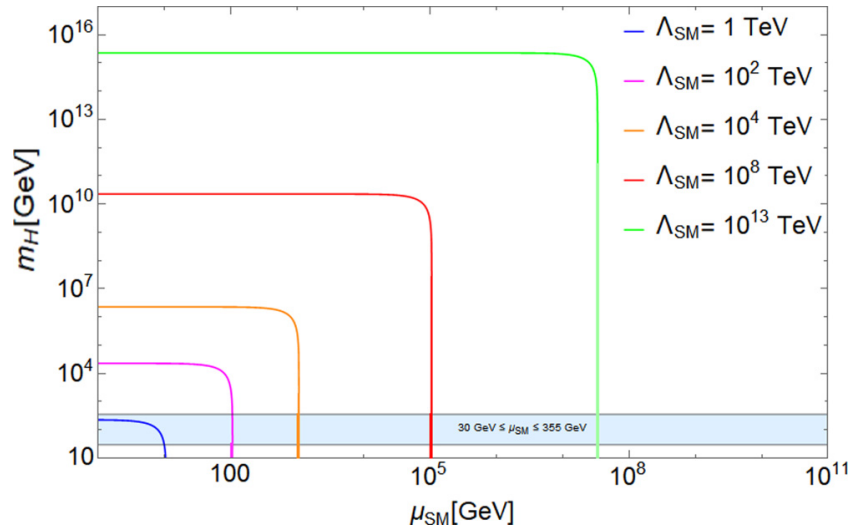

FIG. 2. The value of the SM Higgs mass $m_{H}$ versus SM $\mu_{\mathrm{SM}}$ parameter for theory cutoff values $\Lambda_{\mathrm{SM}}=1,10^{2}, 10^{4}, 10^{8}$, and $10^{13} \mathrm{TeV}$. The anthropic band is shown in blue.

where $\quad m_{H}^{2}$ (tree) $=2 \mu_{\mathrm{SM}}^{2} \quad$ and $\quad \delta m_{H}^{2}=\frac{3}{4 \pi^{2}}\left(-\lambda_{t}^{2}+\frac{g^{2}}{4}+\right.$ $\left.\frac{g^{2}}{8 \cos ^{2} \theta_{W}}+\lambda_{\mathrm{SM}}\right) \Lambda_{\mathrm{SM}}^{2}$, and where $\Lambda_{\mathrm{SM}}$ is the mass scale cutoff beyond which the SM is no longer the appropriate low-energy effective field theory. To gain the measured value of $m_{H} \simeq 125 \mathrm{GeV}$, then for $\Lambda_{\mathrm{SM}} \gg m_{H}$, we can freely tune $m_{H}^{2}$ (tree) to compensate for the large radiative corrections. The situation is shown in Fig. 2 where we show the required value of $\mu_{\mathrm{SM}}$ needed to gain $m_{H}=125 \mathrm{GeV}$ for various choices of $\Lambda_{\mathrm{SM}}$. Since nothing in the SM favors any particular value of $\mu_{\mathrm{SM}}$, we will assume its value is uniformly distributed (logarithmically over the decades of values) in the landscape. It is plain to see that for $\Lambda_{\mathrm{SM}}=1 \mathrm{TeV}$, then a wide range of values for $\mu_{\mathrm{SM}}$ leads to a weak scale (typified here by $m_{H}$ ) within the Agrawal band of allowed values. However, for $\Lambda_{\mathrm{SM}} \gg m_{\text {weak }}$, then only a tiny (finetuned) range of $\mu_{\mathrm{SM}}$ values leads to a viable value for $m_{\mathrm{weak}}$. In this case, stringy naturalness and conventional natural coincide in that an anthropically allowed value of the weak scale requires that the SM be a valid effective field theory only for cutoff value $\Lambda_{\mathrm{SM}} \lesssim 1 \mathrm{TeV}$.

\section{WHY CMSSM/MSUGRA IS LIKELY AN INFREQUENT OCCURRENCE IN THE LANDSCAPE}

In SUSY models, all the quadratic divergences cancel, leaving only logarithmic divergences whose effects may be captured via renormalization group (RG) equations. Thus SUSY models carry with them a solution to the Big hierarchy problem. The question then is: do they carry with them a Little hierarchy problem?

The CMSSM or mSUGRA model [49] is defined by GUT scale input parameters

$$
m_{0}, m_{1 / 2}, A_{0}, \tan \beta, \operatorname{sign}(\mu) \quad(\mathrm{CMSSM} / \mathrm{mSUGRA}),
$$

where $m_{0}$ is the GUT scale unified scalar mass where $m_{H_{u}}=$ $m_{H_{d}}=m_{0}, m_{1 / 2}$ is the unified gaugino mass, $A_{0}$ is the unified trilinear soft breaking term and $\operatorname{sign}(\mu)= \pm 1$. The CMSSM has served for many years as a sort of SUSY paradigm model for SUSY collider and dark matter signal predictions. In CMSSM, the weak-scale soft terms are derived from RG running of the unified soft terms from $Q=m_{\mathrm{GUT}}$ to $Q=m_{\text {weak }}$,

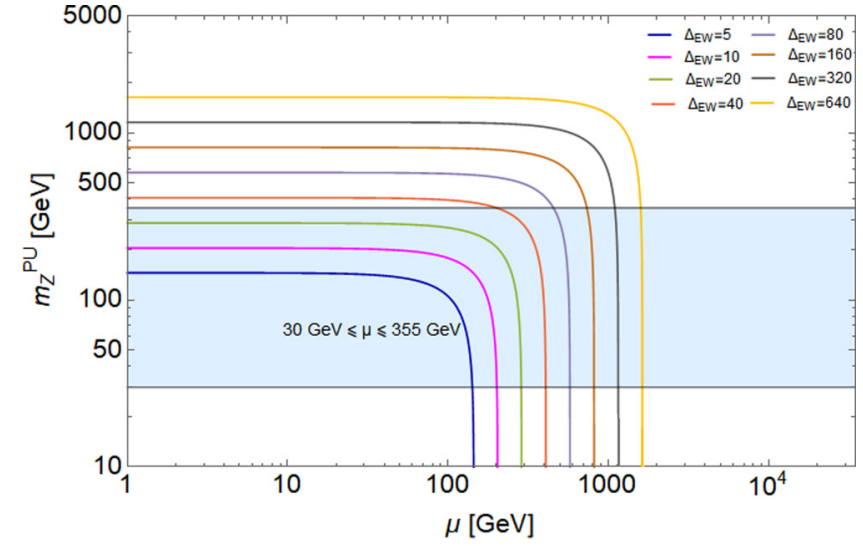

FIG. 3. The pocket universe value of $m_{Z}^{\mathrm{PU}}$ vs the SUSY $\mu$ parameter for various values of EW finetuning parameter $\Delta_{\mathrm{EW}}$. The anthropic band is shown in blue.

where then mixings and mass eigenstates may be evaluated. In the CMSSM model, since $m_{H_{u}}^{2}$ is input at the $m_{\mathrm{GUT}}$ scale, then its weak-scale value is derived. The $\mu$ term is (fine)tuned via Eq. (6) to gain a value in accord with the measured Z-boson mass. ${ }^{5}$

In years past, it was possible to find regions of CMSSM parameter space with small $\mu$ as required for naturalness in the hyperbolic branch [51] or focus point region [52] (HB/FP). The HB/FP region can appear for $m_{0} \lesssim 10 \mathrm{TeV}$ for small values of $A_{0} \sim 0$. However, the measured value of $m_{h} \simeq 125 \mathrm{GeV}$ requires large $A_{t}$ parameter which then moves the HB/FP region out to huge $m_{0}$ values where the $\Sigma_{u}^{u}\left(\tilde{t}_{1,2}\right)$ are large, rendering the model unnatural. Detailed scans of the CMSSM model parameter space in accord with requiring $m_{h}=125 \pm 2 \mathrm{GeV}$ find that the minimal value of $\Delta_{\mathrm{EW}}$ is around 100 but where typically $\Delta_{\mathrm{EW}}$ can range up to $10^{4}$ [16].

To gain some insight on how frequent models with large values of $\Delta_{\mathrm{EW}}$ might occur in the landscape, we take the limit of Eq. (6), wherein the radiative corrections are small so that $m_{Z}^{2} \simeq-2 m_{H_{u}}^{2}-2 \mu^{2}$ and then consider SUSY models where $m_{H_{u}}^{2}$ is driven to large negative values at the weak scale. We can replace $-m_{H_{u}}^{2}$ (weak) by $\Delta_{\mathrm{EW}} \cdot m_{Z}^{2}$ (measured) $/ 2$ to use Eq. (6) to determine the pocket-universe (PU) value of $m_{Z}^{\mathrm{PU}}$ which is expected in SUSY models within the landscape in terms of $\Delta_{\mathrm{EW}}$ and $\mu$.

In Fig. 3, we plot the value of $m_{Z}^{\mathrm{PU}}$ versus $\mu$ for a variety of choices of $\Delta_{\mathrm{EW}}$ ranging from natural values $\sim 5-20$ up to as large as 640 . We also show the shaded band in Fig. 3 which corresponds to values of $m_{Z}^{\mathrm{PU}}$ in accord with the Agrawal et al. allowed values which should give rise to a habitable pocket universe. Assuming a uniform distribution of $\mu$ values in the landscape, then we see from the figure that for low, natural values of $\Delta_{\mathrm{EW}}$ there are significant ranges of $\mu$ which lead to values of $m_{\mathrm{Z}}^{\mathrm{PU}}$ within the anthropic zone. However, as $\Delta_{\mathrm{EW}}$ increases, the expected value of $m_{Z}^{\mathrm{PU}}$ increases well beyond the anthropic allowed zone unless one tunes $\mu$ to lie within a tightening range of (finetuned) values. Thus we would expect

\footnotetext{
${ }^{5}$ We compute SUSY spectra in all models using ISAJET 7.88 [50].
} 
that SUSY models such as CMSSM/mSUGRA-where $\Delta_{\mathrm{EW}}$ cannot attain natural values for $m_{h} \sim 125 \mathrm{GeV}$ - to be rather infrequent occurrences of our fertile patch of the landscape which contains the MSSM as the low-energy effective theory.

\section{RADIATIVE NATURAL SUSY FROM STRINGY NATURALNESS}

From Fig. 3, we see that models with a weak-scale value of the $\mu$ parameter hold a higher likelihood of landing within the narrow band of allowed (pocket universe) weak-scale values from Agrawal et al. [38]. Models with nonuniversal Higgs masses where $m_{0} \neq m_{H_{u}}$ such as the twoextra-parameter nonuniversal Higgs model [53] (NUHM2) or its extension for nonuniversal generations NUHM3 (where $m_{0}(1,2) \neq m_{0}(3)$ as suggested by minilandscape models [54]) are applicable in this situation since the high-scale Higgs masses $m_{H_{u}}^{2}$ and $m_{H_{d}}^{2}$ can be traded for weak-scale inputs $\mu$ and $m_{A}$. Thus the NUHM2 model has input parameters $m_{0}, m_{1 / 2}, A_{0}, \tan \beta, \mu$, and $m_{A}$. The added Higgs mass nonuniversality (which is expected since the Higgs multiplets live in different GUT multiplets from matter scalars) allows for small $\mu \sim 100-300 \mathrm{GeV}$ for any points in model parameter space.

\section{A. Living dangerously}

In Fig. 4, we show the running of the up-Higgs soft mass (actually $\left.\operatorname{sign}\left(m_{H_{u}}^{2}\right) \cdot \sqrt{\left|m_{H_{u}}^{2}\right|}\right)$ in the NUHM2 model for parameters $m_{0}=5 \mathrm{TeV}, m_{1 / 2}=1.2 \mathrm{TeV}, A_{0}=-8 \mathrm{TeV}$ and $\tan \beta=10$ with $m_{H_{d}}=2 \mathrm{TeV}$ but for $m_{H_{u}}(\Lambda)=4,5,6.5$, and $8 \mathrm{TeV}$. We see that for the lower values of $m_{H_{u}}(\Lambda)$, then $m_{H_{u}}^{2}$ runs deeply negative leading to a large negative weak-scale value of $m_{H_{u}}^{2}$ (weak). As $m_{H_{u}}^{2}(\Lambda)$ increases, then it is driven to smaller and smaller (more natural) weak-scale values. For too large a value of $m_{H_{u}}^{2}(\Lambda)$, then its running value is not driven negative at the weak scale and radiative EWSB does not occur. Such unphysical pocket universe solutions must be vetoed. Thus we see that the landscape pull on the soft SUSY

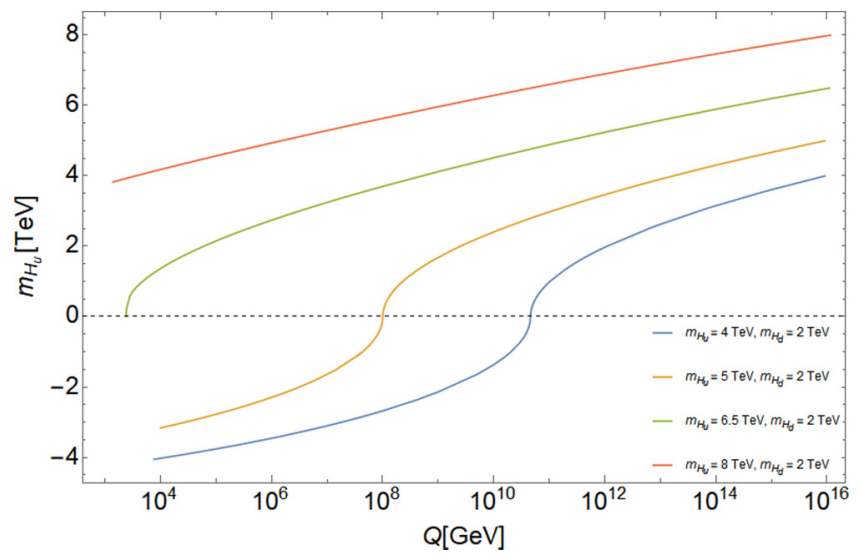

FIG. 4. Running of $m_{H_{u}}^{2}$ vs $Q$ for several choices of $m_{H_{u}}^{2}(\Lambda)$ in the NUHM2 model for $m_{0}=5 \mathrm{TeV}, m_{1 / 2}=1.2 \mathrm{TeV}, A_{0}=-8 \mathrm{TeV}$ and $\tan \beta=10$ for values of $m_{H_{u, d}}$ shown in the figure. The radiatively driven natural SUSY (RNS) case is green. We also show several unnatural SUSY model parameters.

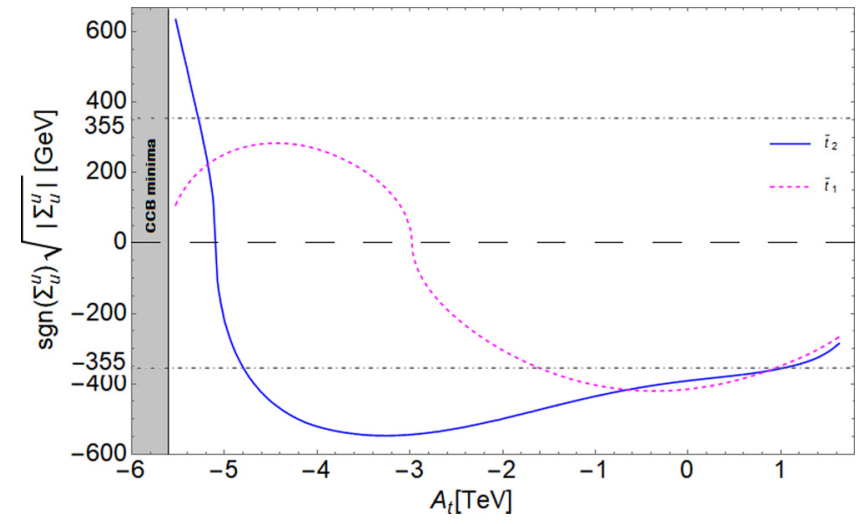

FIG. 5. Contributions $\operatorname{sign}\left(\Sigma_{u}^{u}\right) \sqrt{\left|\Sigma_{u}^{u}\left(\tilde{t}_{1,2}\right)\right|}$ to the weak scale vs $A_{t}$ (weak) in the NUHM2 model with $m_{0}=5 \mathrm{TeV}, m_{1 / 2}=1.2 \mathrm{TeV}$, $m_{A}=2 \mathrm{TeV}, \mu=200 \mathrm{GeV}$, and $\tan \beta=10$.

breaking term $m_{H_{u}}^{2}(\Lambda)$ to large values is just what is needed to gain a natural value of $m_{H_{u}}^{2}$ at the weak scale. This sort of situation has been dubbed as living dangerously in Refs. [55,56] since the soft term is selected to be as large as possible such that EW symmetry is barely broken. ${ }^{6}$ In Refs. [22,23], this situation is called radiatively driven naturalness, or radiative natural SUSY, since the largest viable $m_{H_{u}}^{2}(\Lambda)$ soft terms lead to natural values of $m_{H_{u}}^{2}$ at the weak scale.

A second example of living dangerously within the string landscape is shown in Fig. 5, where we show the contributions $\Sigma_{u}^{u}\left(\tilde{t}_{1,2}\right)$ to the weak scale versus $A_{t}$ for the same NUHM2 parameter choices as in Fig. 4. Here, we see the contribution $\Sigma_{u}^{u}\left(\tilde{t}_{1,2}\right)$ are rather large negative for $A_{0} \sim 0$ with $\operatorname{sign}\left(\Sigma_{u}^{u}\left(\tilde{t}_{1,2}\right)\right) \cdot \sqrt{\left|\Sigma_{u}^{u}\left(\tilde{t}_{1,2}\right)\right|} \sim-400 \mathrm{GeV}$ (in which case we might expect a pocket universe weak scale of $m_{Z}^{\mathrm{PU}} \sim$ $400 \mathrm{GeV})$. As $A_{0}$ moves to large negative values, then we find a point around $A_{0} \sim-5 \mathrm{TeV}$ where both terms become small, yielding contributions to the weak scale which are indeed comparable to our universe's measured value. For much larger (negative) values of $A_{0}$, then we rapidly move into the zone of charge-and-color-breaking (CCB) minima since top squark squared-mass soft terms are driven negative. Thus, in this case, the $A_{0}$ trilinear soft term is statistically preferred to be large (negative) values-but stopping short of CCB minima. This again leads to natural contributions to the weak scale.

A beautiful consequence of the statistical draw to large (negative) $A_{0}$ values is that this induces large mixing in the top-squark sector, which also ends up maximizing the value of $m_{h}[20,21]$. The case here is shown in Fig. 6 where we plot the light Higgs mass $m_{h}$ versus $A_{0}$ for the same parameters as in Fig. 5. For an unnatural value of $A_{0} \sim 0$, then we expect $m_{h} \sim$ $119 \mathrm{GeV}$. However, as $A_{0}$ increases to large (negative) values, then $m_{h} \rightarrow 124-126 \mathrm{GeV}$, in accord with its measured value in our universe. A third example occurs if we allow the

\footnotetext{
${ }^{6}$ Arkani-Hamed and Dimopoulos [55] state: "Anthropic reasoning leads to the conclusion that we live dangerously close to violating an important but fragile feature of the low-energy world...," in this case, appropriate electroweak symmetry breaking.
} 


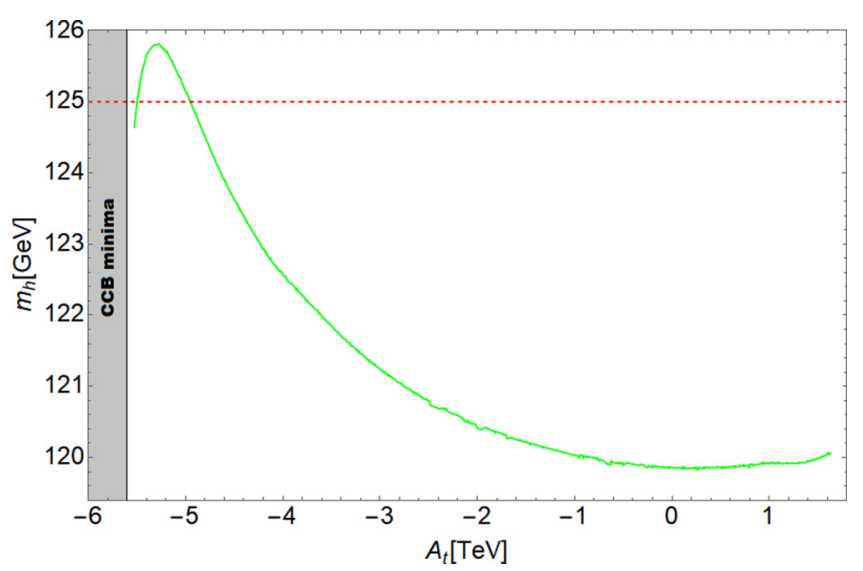

FIG. 6. Value of $m_{h}$ vs $A_{t}$ in the NUHM2 model with $m_{0}=$ $5 \mathrm{TeV}, m_{1 / 2}=1.2 \mathrm{TeV}, m_{A}=2 \mathrm{TeV}, \mu=200 \mathrm{GeV}$, and $\tan \beta=10$.

landscape to statistically select large values of $m_{1 / 2}$. This is shown in Fig. 7(a) where we plot $\sqrt{\left|\Sigma_{u}^{u}\left(\tilde{t}_{1,2}\right)\right|}$ versus $m_{1 / 2}$ for fixed $m_{0}=5 \mathrm{TeV}$ and other parameters fixed as in the Caption. In this case, then as the gaugino masses increase, especially the gluino mass, then large $S U(3)_{C}$ contributions $M_{3}$ to the top-squark soft masses tend to drive them to large values, thus increasing the $\Sigma_{u}^{u}\left(\tilde{t}_{1,2}\right)$ contributions to values well beyond our measured value of the weak scale. In such cases, we would expect too large a value of the weak scale, typically in violation of Agrawal et al. bounds. These would lead to violations of the so-called "atomic principle," and atoms as we know them would not form with too large a value of $m_{\text {weak }}$. In Fig. 7(b), we show the values of $\Sigma_{u}^{u}\left(\tilde{t}_{1,2}\right)$ versus $m_{0}$ for fixed $m_{1 / 2}=1.2 \mathrm{TeV}$. In this case, if the scalar soft terms become too large, then again the values of $\sqrt{\left|\Sigma_{u}^{u}\left(\tilde{t}_{1,2}\right)\right|}$ become too large and we would gain a pocket universe weakscale value in excess of bounds from nuclear/atomic anthropic requirements.

\section{B. Naturalness versus stringy naturalness}

Next, we would like to explore how the notion of stringy naturalness compares to conventional naturalness measures.
To begin, we plot naturalness contours in Fig. 8(a) in the $m_{0}$ versus $m_{1 / 2}$ plane of the CMSSM/mSUGRA model for $A_{0}=$ $0, \tan \beta=10$ and $\mu>0$. The $\Delta_{\mathrm{HS}}<100$ contour shows the region of lighter top squarks for small $A_{0}$ parameter in the low $m_{0}$, low $m_{1 / 2}$ region. The orange contour denotes where $\Delta_{\mathrm{BG}}<30$ while the green contour denotes where $\Delta_{\mathrm{EW}}<$ 30. These latter two measure reflect focus point behavior in that $\mathrm{TeV}$-scale stops are still natural (since the contours are relatively flat with variation in $m_{0}$ ). The LHC limit on $m_{\tilde{g}}>2.25 \mathrm{TeV}$ is shown as the magenta contour. This plane might lead to skepticism regarding weak-scale SUSY since the LHC allowed region is so far beyond the naturalness upper bounds. Also, in this plane, the light Higgs mass $m_{h}$ is always below $123 \mathrm{GeV}$. The important lesson for now is that the more natural regions occur at the lowest $m_{0}$ and $m_{1 / 2}$ values, where the various measures are smallest, and the sparticle masses are closest to the measured weak scale.

For comparison, we show the same $m_{0}$ versus $m_{1 / 2}$ plane in Fig. 8(b), but this time for the NUHM2 model where $\mu=200 \mathrm{GeV}, m_{A}=2 \mathrm{TeV}, A_{0}=-1.6 m_{0}$ and as before $\tan \beta=10$. In this case, the lower left region of the plot actually leads to CCB minima (blue contour) so that $\Delta_{\mathrm{HS}}$ is not computable. $\Delta_{\mathrm{BG}}$ is still computable and has shrunk down into the lower-left due to large $A_{0}$ term contributions. However, we now see that the $\Delta_{\mathrm{EW}}$ values have expanded out to much larger $m_{0}$ and $m_{1 / 2}$ values since it is largely determined by the $\Sigma_{u}^{u}\left(\tilde{t}_{1,2}\right)$ values, since $\mu$ is fixed to be near the measured EW scale. Here, a substantial amount of natural SUSY parameter space lies beyond LHC gluino mass limits. Nonetheless, the lower portions of $m_{0}, m_{1 / 2}$ space are more natural since they yield smaller values of $\Delta_{\mathrm{EW}}$. Also, we show contours of $m_{h}=123$ and $127 \mathrm{GeV}$. The region with $m_{h}=125 \pm 2 \mathrm{GeV}$ overlaps nicely with the natural SUSY region, with plenty of parameter space beyond the LHC gluino mass limit.

Now we would like to compare the previous natural SUSY regions against the regions preferred by Douglas' stringy naturalness. To accomplish this, next we show again in Figs. 9(a)9 (d) the $m_{0}$ versus $m_{1 / 2}$ plane for the NUHM2 model with the same parameters as in Fig. 8(b). We generate SUSY soft parameters in accord with Eq. (7) for various values of
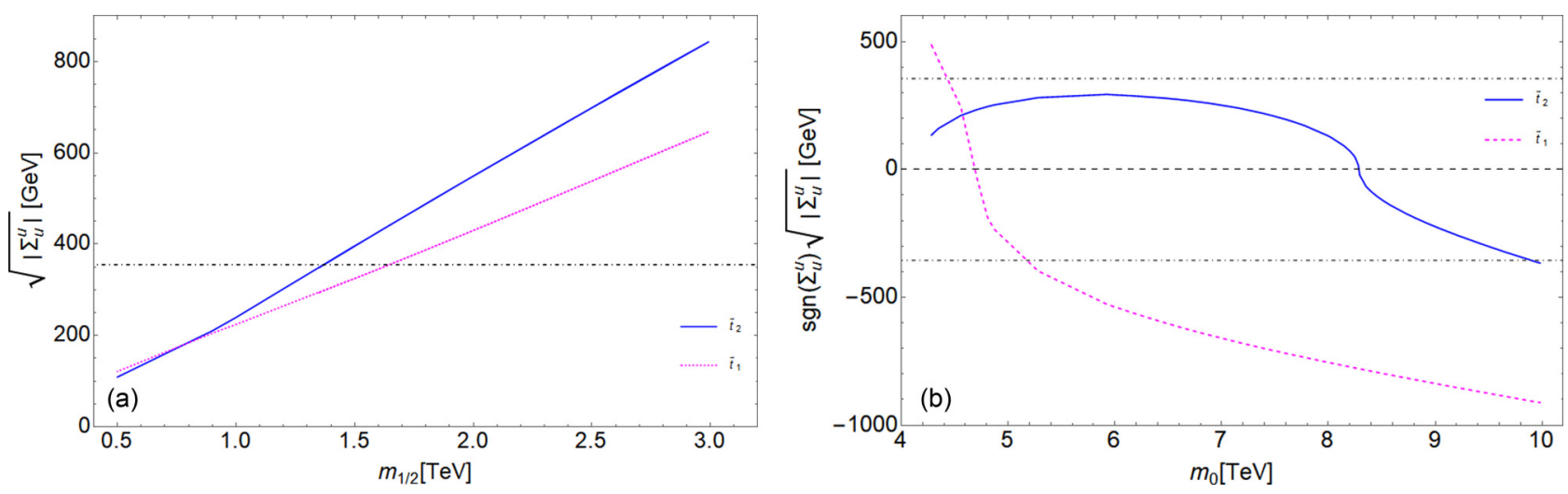

FIG. 7. Plot of $\sqrt{\left|\sum_{u}^{u}\left(\tilde{t}_{1,2}\right)\right|}$ vs (a) $m_{1 / 2}$ for $m_{0}=5 \mathrm{TeV}$ and (b) $m_{0}$ for $m_{1 / 2}=1.2 \mathrm{TeV}$ in the NUHM 2 model. We also take $A_{0}=-8 \mathrm{TeV}$, $\mu=200 \mathrm{GeV}, m_{A}=2 \mathrm{TeV}$, and $\tan \beta=10$. The horizontal lines shows where contributions to $m_{\mathrm{weak}}$ exceed a factor four times its measured value in our pocket universe. 

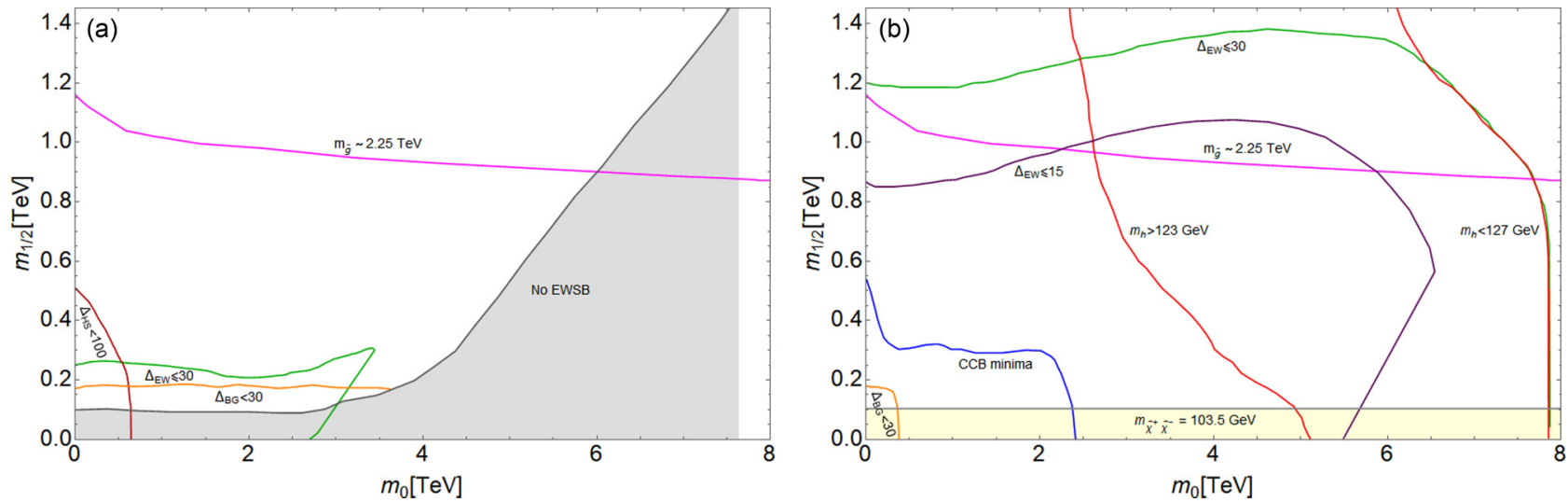

FIG. 8. The $m_{0}$ vs $m_{1 / 2}$ plane of (a) the mSUGRA/CMSSM model with $A_{0}=0$ and (b) the NUHM 2 model with $A_{0}=-1.6 m_{0}, \mu=$ $200 \mathrm{GeV}$, and $m_{A}=2 \mathrm{TeV}$. For both cases, we take $\tan \beta=10$. We show contours of various finetuning measures along with Higgs mass contours and LEP2 and LHC Run 2 search limits.

$n=2 n_{F}+n_{D}-1=1,2,3$, and $4 .^{7}$ The more stringy natural regions of parameter space are denoted by the higher density of sampled points.

${ }^{7}$ The high $n$ values allow for a consistent sampling of NUHM2 parameter space since here we fix the $A_{0}$ parameter in terms of $m_{0}$ so it never gets too large (which would lead to CCB minima and nonanthropic vacua) as compared with Ref. [40].
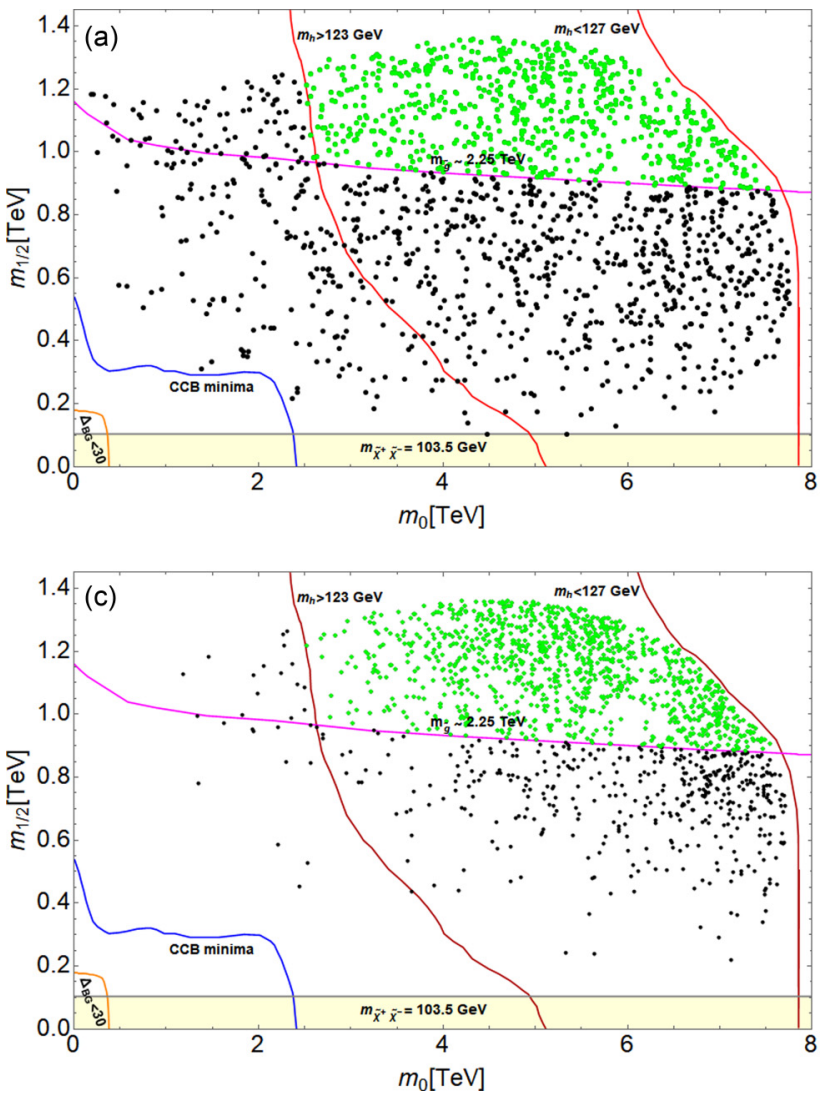

In Fig. 9(a), we show the stringy natural regions for the case of $n=1$. Of course, no dots lie below the CCB boundary since such minima must be vetoed as they likely lead to an unlivable pocket universe. Beyond the CCB contour, the solutions are in accord with livable vacua. However, now the density of points increases with increasing $m_{0}$ and $m_{1 / 2}$ (linearly, for $n=1$ ), showing that the more stringy natural regions lie at the highest $m_{0}$ and $m_{1 / 2}$ values which are consistent with generating a weak scale within the Agrawal bounds. Beyond these bounds, the density of points of course drops to
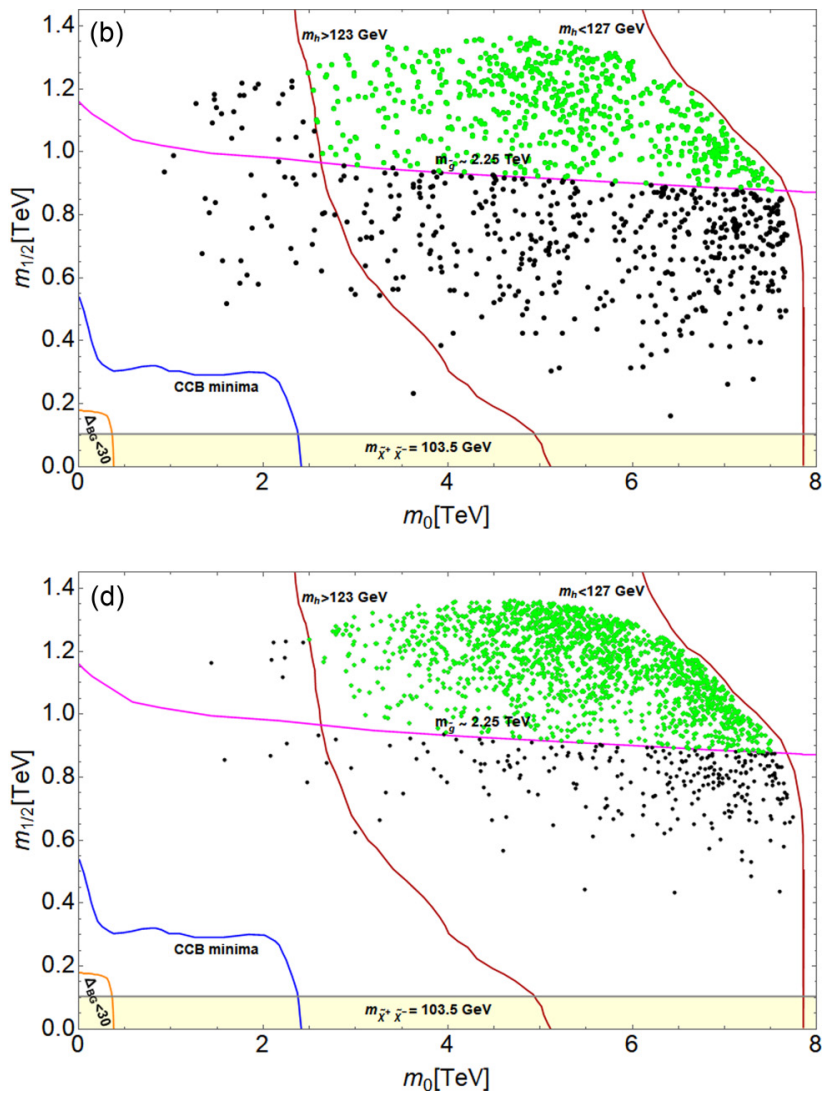

FIG. 9. The $m_{0}$ vs $m_{1 / 2}$ plane of the NUHM2 model with $A_{0}=-1.6 m_{0}, \mu=200 \mathrm{GeV}$, and $m_{A}=2 \mathrm{TeV}$ and (a) an $n=1 \mathrm{draw}$ on soft terms, (b) an $n=2$ draw, (c) an $n=3$ draw, and (d) an $n=4$ draw. The higher density of points denotes greater stringy naturalness. The LHC Run 2 limit on $m_{\tilde{g}}>2.25 \mathrm{TeV}$ is shown by the magenta curve. The lower yellow band is excluded by LEP2 chargino pair search limits. 
zero since contributions to the weak scale exceed its measured value by a factor 4 . There is some fluidity of this latter bound as indicated in Fig. 1, so values of $\Delta_{\mathrm{EW}} \sim 20-40$ might also be entertained The result that stringy naturalness for $n \geqslant 1$ favors the largest soft terms (subject to $m_{Z}^{\mathrm{PU}}$ not ranging too far from our measured value) stands in stark contrast to conventional naturalness which favors instead the lower values of soft terms. Needless to say, the stringy natural favored region of parameter space is in close accord with LHC results in that LHC find $m_{h}=125 \mathrm{GeV}$ with no sign yet of sparticles.

In Figs. 9(b)-9(d), we show the same $m_{0}$ versus $m_{1 / 2}$ planes but for $n=2,3$, and 4 . As $n$ steadily increases, the stringy natural region is pushed more strongly to large values of $m_{0}$ and $m_{1 / 2}$ so that relatively few vacua lie below the LHC gluino mass limit. Indeed, we would say that the stringy naturalness prediction is that LHC should see a Higgs mass around $125 \mathrm{GeV}$ with no sign yet of sparticles.

\section{WHY HIGH-SCALE SUSY IS LIKELY A RARE OCCURRENCE IN THE LANDSCAPE}

While it is often argued that the landscape opens new territory in model building and motivation for finetuned SUSY models, here we will argue that such models should be, while possible, relatively rare occurrences on the string theory landscape. The rationale is usually that since the cosmological constant is finetuned, then perhaps a similar mechanism allows for a finetuned weak scale. Here we would counter with two observations. First, in Weinberg's approach, the cosmological constant is about as natural as possible subject to allowing for pocket universes that allow for galaxy condensation. Second, there is at present no plausible alternative for a tiny cosmological constant other than the landscape selection. As alternatives to various high-scale SUSY models described below, SUSY with radiatively driven naturalness should be a relatively common occurrence on the landscape, as argued above, whereas finetuned models, while possible, should be relatively rare.

(1) Split SUSY. Split SUSY was proposed in the aftermath of the emergence of the landscape as a model which retained the desirable SUSY model feature of gauge coupling unification and a WIMP dark matter candidate whilst eschewing the requirement of weak-scale naturalness. In this approach, then, SUSY scalar masses other than the SM Higgs can range from $\sim 10^{3} \mathrm{TeV}$ up to possibly $10^{8} \mathrm{TeV}[32,57,58]$. Meanwhile, gauginos and higgsinos are highly split from the scalars and can occupy masses in the $0.1-10 \mathrm{TeV}$ range. This type of split spectrum maintains gauge coupling unification and a mixed gaugino-higgsino type WIMP. The ultraheavy scalars suppress flavor- and CP-violating processes and thus explain the lack of such effects in experiments. Recent work comparing split SUSY models with the measured value of $m_{h} \simeq 125 \mathrm{GeV}$ favors a lower range of scalar masses $\tilde{m} \sim$ $10-10^{4} \mathrm{TeV}$ [59]. Upon integrating out the supermassive scalar particles, then the low-energy effective theory [60] contains SM particles plus the gauginos and higgsinos. Thus quadratic divergences should give rise to models as in Fig. 2 with a scale $\Lambda \sim \tilde{m}$, i.e., the required finetuning leads to a rare occurrence on the landscape for uniformly distributed values of $\mu_{\mathrm{SM}}^{\mathrm{eff}}$.
(2) High-scale SUSY. In high-scale (HS) SUSY, one again discards naturalness in the hope that the landscape will solve the Big hierarchy problem. However, for HS SUSY, one allows all the SUSY partners to obtain large masses. The energy scale for HS SUSY may be labeled as $\Lambda_{\mathrm{HS}}$ and values considered in the literature range from $10^{2}-10^{9} \mathrm{TeV}$ [33]. Thus the matching conditions between the MSSM and the SM effective field theories are implemented around the scale $\Lambda_{\mathrm{HS}}$. The resultant Higgs mass dependence on $\mu_{\mathrm{SM}}$ will be as in Fig. 2 so again we would expect HS SUSY to be a rare occurrence on the landscape as compared to RNS.

(3) PeV SUSY. In PeV SUSY [34], SUSY breaking occurs via a charged (i.e., nonsinglet) hidden sector $F$ term leading to scalar masses at the PeV scale $\left(1 \mathrm{PeV}=10^{3} \mathrm{TeV}\right)$, while gaugino soft breaking terms are suppressed and hence dominated by the anomaly-mediation form so that the wino turns out to be LSP. A $2-3 \mathrm{TeV}$ wino is suggested to gain accord with the measured dark matter density which in turn suggests $m_{3 / 2} \sim \tilde{m} \sim \mathrm{PeV}$. Some motivation for the PeV scale also comes from the neutrino sector by assuming neutrinos charged under an additional $U(1)^{\prime}$ so that Dirac neutrino masses arise from non-renormalizable operators. The light Higgs mass is expected at $m_{h} \sim 125-145 \mathrm{GeV}$. The PeV SUSY models would correspond to curves in Fig. 2 with $\Lambda \sim 10^{3} \mathrm{TeV}$. Thus we would expect PeV SUSY to be relatively rare on the landscape.

(3) Spread SUSY. In Spread SUSY [35], three scales of sparticles occur. The first possibility is that scalar masses occur at $\tilde{m} \sim 10^{6} \mathrm{TeV}$ with gauginos at $10^{2} \mathrm{TeV}$ and higgsinos around $1 \mathrm{TeV}$ in order to gain accord with the measured dark matter abundance. A second possibility considered has scalars around $\tilde{m} \sim 10^{3} \mathrm{TeV}$ with higgsinos an order of magnitude lower and gauginos at the $\mathrm{TeV}$ scale (where winos with mass $\sim 3 \mathrm{TeV}$ would saturate the measured dark matter abundance). Thus Spread SUSY models would correspond to curves from Fig. 2 with $\Lambda \sim 10^{3}-10^{6} \mathrm{TeV}$. We would thus expect Spread SUSY to be rare on the string landscape.

(4) Minisplit. Minisplit SUSY [36] emerged after the LHC Higgs discovery and attempted to reconcile Split SUSY with a Higgs boson mass of $m_{h} \simeq 125 \mathrm{GeV}$. To accommodate the Higgs mass, the mass scale of the heavy scalars was decreased to $(1) \tilde{m} \sim 10^{2} \mathrm{TeV}$ scale for a heavy $\mu \sim 10^{2} \mathrm{TeV}$ model with TeV-scale gauginos (light AMSB) or (2) $\tilde{m} \sim 10^{4} \mathrm{TeV}$ with $10^{2} \mathrm{TeV}$ gauginos but with small $\mu<\mathrm{TeV}$ (heavy AMSB). Models with $U(1)^{\prime}$ mediation were also considered. The minisplit models would thus correspond to curves in Fig. 2 with $\Lambda \sim 10^{2}-10^{4} \mathrm{TeV}$. These models should be rare on the landscape, but less rare than original Split SUSY models.

\section{IMPLICATIONS OF STRINGY NATURALNESS FOR COLLIDER AND DARK MATTER SEARCHES}

In light of the implications of stringy naturalness, how then ought SUSY to be revealed at collider and dark matter search experiments? Since the superpotential $\mu$ parameter must not be too far removed from our measured value of the weak scale, then higgsinos must also be light. A compelling signature emerges from $\tilde{\chi}_{1}^{0} \tilde{\chi}_{2}^{0}$ production at LHC $[61,62]$ where $\tilde{\chi}_{2}^{0} \rightarrow$ $\tilde{\chi}_{1}^{0} \ell^{+} \ell^{-}$in recoil against a hard radiated initial state jet. The 
soft dilepton plus jet + MET signal should emerge slowly as more and more data accrues at LHC. ${ }^{8}$ Meanwhile, more conventional SUSY signatures such as those from gluino or top squark pair production might be visible at HL-LHC [64], but also may require an upgrade to HE-LHC since gluinos can range up to $m_{\tilde{g}} \lesssim 6 \mathrm{TeV}$ while stops are required at $m_{\tilde{t}_{1}} \lesssim$ $3 \mathrm{TeV}[65,66]$. Same-sign diboson signatures from wino pair production are an additional possibility $[67,68]$.

The required light higgsinos within the natural mass range $m$ (higgsinos) $\sim 100-300 \mathrm{GeV}$ provide a lucrative target for an ILC-type $e^{+} e^{-}$collider with $\sqrt{s}>2 m$ (higgsino). Such a machine would act as a higgsino factory where the various higgsino masses could be precisely determined. The higgsino mass splittings are sensitive to the (heavier) gaugino masses; consequent fits to gaugino masses could then allow for tests of hypotheses regarding gaugino mass unification [69].

With regard to dark matter searches, we remark that naturalness in the QCD sector requires the PQ solution to the strong $\mathrm{CP}$ problem and the concommitant axion $a$ [70]. To ensure $\bar{\theta} \lesssim 10^{-10}$, then the SUSY axion model must be safe from gravity corrections. This can occur when both PQ symmetry and $R$-parity conservation emerge from underlying discrete $R$ symmetries, such as the recent example of $\mathbb{Z}_{24}^{R}$ [46]. In this case, one gains a solution to the SUSY $\mu$ problem [45] via a Kim-Nilles operator leading to a SUSY DFSZ axion. While the SUSY DFSZ axion has suppressed couplings to photons (and may not be observable with present technology [71]) the thermally underproduced higgsinolike WIMPs, which make up typically only about $10 \%$ of dark matter, should ultimately be detectable via multiton noble liquid detectors [72].

\section{CONCLUSIONS}

In this paper, we have explored Douglas' notion of stringy naturalness - that the value of an observable $\mathcal{O}_{2}$ is more natural than $\mathcal{O}_{1}$ if the number of phenomenologically viable vacua giving rise to $\mathcal{O}_{2}$ is great than the number of vacua giving rise to $\mathcal{O}_{1}$ - and its relation to conventional naturalness, with regard to the big and little hierarchy problems and why the value of the weak scale $m_{\text {weak }}$ in our pocket universe is only $m_{\text {weak }} \sim$ $100 \mathrm{GeV}$. We interpret phenomenologically viable to mean a fertile patch of string theory vacua leading to the SM as the low-energy effective theory with a value for the weak scale not too far (a factor four) beyond our measured value, as required by the nuclear physics calculations of Agrawal et al. [38].

It is often claimed that the string theory landscape picture allows us to eschew the common notion of naturalness in that certain parameters, such as the cosmological constant or the magnitude of the weak scale, are environmentally determined. With regard to the SM, since there appears to be no theoretical preference for any value of Higgs potential parameter $\mu_{\mathrm{SM}}$, we would expect it to be roughly uniformly distributed across the decades of possibilities. For the SM to be valid up to scales $\Lambda_{\mathrm{SM}} \gg m_{\text {weak }}$, only tiny permissible ranges of $\mu_{\mathrm{SM}}$ could give rise to weak-scale values leading to livable pocket universes (Fig. 2). In contrast, for effective

\footnotetext{
${ }^{8}$ Excess events of this type seem to be emerging already in a recent Atlas analysis with $139 \mathrm{fb}^{-1}$ [63].
}

theories like the MSSM, then since quadratic divergences all cancel, there appears to exist large swaths of superpotential $\mu$ values leading to weak scales at $\sim 100 \mathrm{GeV}$. Thus we conclude that stringy naturalness would favor the MSSM over the SM as the appropriate low-energy effective field theory, in agreement with, and not opposed to, conventional notions of naturalness.

We also explored implications of stringy naturalness for various SUSY models. The CMSSM (mMSUGRA) model with $m_{h} \sim 125 \mathrm{GeV}$-where $\Delta_{\mathrm{EW}}$ is found to be $\sim 10^{2}-10^{4}$ - should be rather infrequent on the landscape since only tiny ranges of $\mu$ values lead to $m_{\text {weak }} \sim 100 \mathrm{GeV}$. Likewise, the panoply of SUSY models with scalar masses in the $10^{2}-10^{11} \mathrm{TeV}$ range (Split SUSY, HS SUSY, PeV SUSY, Spread SUSY, and Minisplit SUSY) all appear infrequent on the landscape due to the unlikelihood of vacua which have unrelated parameters compensating for overly large contributions to the weak scale. This is in spite of the rather general expectation that soft terms should be statistically selected for large values, as expected in stringy models with multiple hidden sectors.

The statistical draw to large soft terms is just what is needed for SUSY with radiatively-driven naturalness, where one is living dangerously in that if the soft terms are much larger, then one is placed into CCB or no EWSB vacua (which must be anthropically vetoed). This situation, that soft terms are expected as large as possible such that EW symmetry is barely broken and that all independent contributions to the weak scale are within a factor four of our measured value, is just what is needed to radiatively drive the soft terms to natural values.

In these radiative natural SUSY (RNS) models, we further compared the locus of stringy natural regions of parameter space in the $m_{0}$ versus $m_{1 / 2}$ plane to the conventionally natural regions. Here, there is a major difference: conventional naturalness favors soft terms as close to the $100 \mathrm{GeV}$ scale as possible while stringy naturalness favors soft terms as large as possible such that the weak scale is not too far removed from our measured value of $\sim 100 \mathrm{GeV}$. We can read off from Fig. 9 that stringy naturalness predicts a Higgs mass $m_{h} \sim 125 \mathrm{GeV}$ whilst sparticles remain (at present) beyond LHC reach. Needless to say, this postdiction has been verified by Run 2 data from LHC13.

The stringy natural RNS SUSY model gives rise to specific tests at collider and dark matter search experiment. We expect the soft dilepton plus jet plus MET signature to slowly emerge at HL-LHC as more and more integrated luminosity accrues, while gluino, top squark and wino pair production signals might require a HE-LHC for discovery. Stringy naturalness cries out for construction of an ILC $e^{+} e^{-}$collider with $\sqrt{s}>2 m$ (higgsino) which would act as a higgsino factory. We still expect WIMPs at multi-ton noble liquid dark matter detection experiments but SUSY DFSZ axions will likely be difficult to detect.

\section{ACKNOWLEDGMENTS}

This work was supported in part by the US Department of Energy, Office of High Energy Physics. The work of H.B. was performed at the Aspen Center for Physics, which is supported by National Science Foundation under Grant No. PHY-1607611. 
[1] L. Susskind, Dynamics of spontaneous symmetry breaking in the weinberg-salam theory, Phys. Rev. D 20, 2619 (1979).

[2] E. Witten, Dynamical breaking of supersymmetry, Nucl. Phys. B 188, 513 (1981); R. K. Kaul, Gauge Hierarchy in a Supersymmetric Model, Phys. Lett. B 109, 19 (1982).

[3] H. Baer and X. Tata, Weak Scale Supersymmetry: From Superfields to Scattering Events (University Press, Cambridge, UK, 2006), p. 537; for a recent review, see, e.g., D. J. H. Chung, L. L. Everett, G. L. Kane, S. F. King, J. D. Lykken, and L. T. Wang, The Soft supersymmetry breaking Lagrangian: Theory and applications, Phys. Rept. 407, 1 (2005).

[4] E. Aprile et al. (XENON Collaboration), Dark Matter Search Results from a One Ton-Year Exposure of XENON1T, Phys. Rev. Lett. 121, 111302 (2018); X. Cui et al. (PandaX-II Collaboration), Dark Matter Results From 54-Ton-Day Exposure of PandaX-II Experiment, ibid. 119, 181302 (2017); D. S. Akerib et al. (LUX Collaboration), Results from a Search for Dark Matter in the Complete LUX Exposure, ibid. 118, 021303 (2017).

[5] M. Aaboud et al. (ATLAS Collaboration), Search for squarks and gluinos in final states with jets and missing transverse momentum using $36 \mathrm{fb}^{-1}$ of $\sqrt{s}=13 \mathrm{TeV}$ pp collision data with the ATLAS detector, Phys. Rev. D 97, 112001 (2018).

[6] A. M. Sirunyan et al. (CMS Collaboration), Search for top squark pair production in pp collisions at $\sqrt{s}=13 \mathrm{TeV}$ using single lepton events, J. High Energy Phys. 10 (2017) 019; The ATLAS collaboration (ATLAS Collaboration), "Search for direct top squark pair production in the 3-body decay mode with a final state containing one lepton, jets, and missing transverse momentum in $\sqrt{s}=13 \mathrm{TeV} p p$ collision data with the ATLAS detector," ATLAS-CONF-2019-017.

[7] J. R. Ellis, K. Enqvist, D. V. Nanopoulos, and F. Zwirner, Observables in low-energy superstring models, Mod. Phys. Lett. A 01, 57 (1986).

[8] R. Barbieri and G. F. Giudice, Upper bounds on supersymmetric particle masses, Nucl. Phys. B 306, 63 (1988).

[9] S. Dimopoulos and G. F. Giudice, Naturalness constraints in supersymmetric theories with nonuniversal soft terms, Phys. Lett. B 357, 573 (1995).

[10] G. W. Anderson and D. J. Castano, Challenging weak scale supersymmetry at colliders, Phys. Rev. D 53, 2403 (1996).

[11] D. Kim, P. Athron, C. Balázs, B. Farmer, and E. Hutchison, Bayesian naturalness of the CMSSM and CNMSSM, Phys. Rev. D 90, 055008 (2014); P. Athron, C. Balazs, B. Farmer, A. Fowlie, D. Harries, and D. Kim, Bayesian analysis and naturalness of (Next-to-)Minimal Supersymmetric Models, J. High Energy Phys. 10 (2017) 160.

[12] R. Barbieri and A. Strumia, About the fine tuning price of LEP, Phys. Lett. B 433, 63 (1998).

[13] H. Baer, V. Barger, S. Salam, H. Serce, and K. Sinha, LHC SUSY and WIMP dark matter searches confront the string theory landscape, J. High Energy Phys. 04 (2019) 043.

[14] H. Baer, V. Barger, and D. Mickelson, How conventional measures overestimate electroweak fine-tuning in supersymmetric theory, Phys. Rev. D 88, 095013 (2013).

[15] A. Mustafayev and X. Tata, Supersymmetry, Naturalness, and Light Higgsinos, Indian J. Phys. 88, 991 (2014).

[16] H. Baer, V. Barger, D. Mickelson, and M. Padeffke-Kirkland, SUSY models under siege: LHC constraints and electroweak fine-tuning, Phys. Rev. D 89, 115019 (2014).
[17] H. Baer, V. Barger, and M. Savoy, Supergravity gauge theories strike back: There is no crisis for SUSY but a new collider may be required for discovery, Phys. Scr. 90, 068003 (2015).

[18] M. Papucci, J. T. Ruderman, and A. Weiler, Natural SUSY endures, J. High Energy Phys. 09 (2012) 035.

[19] C. Brust, A. Katz, S. Lawrence, and R. Sundrum, SUSY, the third generation and the LHC, J. High Energy Phys. 03 (2012) 103.

[20] M. Carena and H. E. Haber, Higgs boson theory and phenomenology, Prog. Part. Nucl. Phys. 50, 63 (2003); P. Draper and H. Rzehak, A review of higgs mass calculations in supersymmetric models, Phys. Rept. 619, 1 (2016).

[21] H. Baer, V. Barger, and A. Mustafayev, Implications of a $125 \mathrm{GeV}$ Higgs scalar for LHC SUSY and neutralino dark matter searches, Phys. Rev. D 85, 075010 (2012).

[22] H. Baer, V. Barger, P. Huang, A. Mustafayev, and X. Tata, Radiative Natural SUSY with a $125 \mathrm{GeV}$ Higgs Boson, Phys. Rev. Lett. 109, 161802 (2012).

[23] H. Baer, V. Barger, P. Huang, D. Mickelson, A. Mustafayev, and X. Tata, Radiative natural supersymmetry: Reconciling electroweak fine-tuning and the Higgs boson mass, Phys. Rev. D 87, 115028 (2013).

[24] H. Baer, V. Barger, and M. Savoy, Upper bounds on sparticle masses from naturalness or how to disprove weak scale supersymmetry, Phys. Rev. D 93, 035016 (2016).

[25] H. Baer, V. Barger, J. S. Gainer, P. Huang, M. Savoy, H. Serce, and $\mathrm{X}$. Tata, What hadron collider is required to discover or falsify natural supersymmetry? Phys. Lett. B 774, 451 (2017).

[26] H. Baer, V. Barger, M. Padeffke-Kirkland, and X. Tata, Naturalness implies intra-generational degeneracy for decoupled squarks and sleptons, Phys. Rev. D 89, 037701 (2014).

[27] S. Weinberg, Anthropic Bound on the Cosmological Constant, Phys. Rev. Lett. 59, 2607 (1987); The cosmological constant problem, Rev. Mod. Phys. 61, 1 (1989).

[28] R. Bousso and J. Polchinski, Quantization of four form fluxes and dynamical neutralization of the cosmological constant, J. High Energy Phys. 06 (2000) 006.

[29] L. Susskind, The Anthropic landscape of string theory, in Universe or multiverse? edited by Bernard Carr (Cambridge University Press, 2007).

[30] M. R. Douglas, Statistical analysis of the supersymmetry breaking scale, arXiv:hep-th/0405279.

[31] S. Ashok and M. R. Douglas, Counting flux vacua, J. High Energy Phys. 01 (2004) 060.

[32] N. Arkani-Hamed and S. Dimopoulos, Supersymmetric unification without low energy supersymmetry and signatures for fine-tuning at the LHC, J. High Energy Phys. 06 (2005) 073.

[33] V. Barger, C. W. Chiang, J. Jiang, and T. Li, Axion models with high-scale supersymmetry breaking, Nucl. Phys. B 705, 71 (2005); V. Barger, J. Jiang, P. Langacker, and T. $\mathrm{Li}$, Non-canonical gauge coupling unification in high-scale supersymmetry breaking, ibid. 726, 149 (2005); C. Liu, A supersymmetry model of leptons, Phys. Lett. B 609, 111 (2005); Supersymmetry for fermion masses, Commun. Theor. Phys. 47, 835 (2007); C. Liu and Z. H. Zhao, $\theta_{13}$ and the Higgs mass from high-scale supersymmetry, ibid. 59, 467 (2013); G. Elor, H. S. Goh, L. J. Hall, P. Kumar, and Y. Nomura, Environmentally selected wimp dark matter with high-scale supersymmetry breaking, Phys. Rev. D 81, 095003 (2010); R. Sato, S. Shirai, and K. Tobioka, Gluino decay as a probe of high scale 
supersymmetry breaking, J. High Energy Phys. 11 (2012) 041; J. Hisano, K. Ishiwata, and N. Nagata, Direct search of dark matter in high-scale supersymmetry, Phys. Rev. D 87, 035020 (2013); J. Hisano, T. Kuwahara, and N. Nagata, Grand unification in high-scale supersymmetry, Phys. Lett. B 723, 324 (2013); I. Masina and M. Quiros, On the veltman condition, the hierarchy problem and high-scale supersymmetry, Phys. Rev. D 88, 093003 (2013); N. Nagata and S. Shirai, Higgsino dark matter in high-scale supersymmetry, J. High Energy Phys. 01 (2015) 029; S. A. R. Ellis and J. D. Wells, High-scale supersymmetry, the Higgs boson mass, and gauge unification, Phys. Rev. D 96, 055024 (2017); E. Dudas, T. Gherghetta, Y. Mambrini, and K. A. Olive, Inflation and high-scale supersymmetry with an EeV gravitino, ibid. 96, 115032 (2017); S. A. R. Ellis, T. Gherghetta, K. Kaneta, and K. A. Olive, New weak-scale physics from $\mathrm{SO}(10)$ with high-scale supersymmetry, ibid. 98, 055009 (2018); K. Y. Choi and H. M. Lee, Axino abundances in high-scale supersymmetry, Phys. Dark Univ. 22, 202 (2018); E. Dudas, T. Gherghetta, K. Kaneta, Y. Mambrini, and K. A. Olive, Limits on $R$-parity violation in high-scale supersymmetry, Phys. Rev. D 100, 035004 (2019).

[34] J. D. Wells, PeV-scale supersymmetry, Phys. Rev. D 71, 015013 (2005).

[35] L. J. Hall and Y. Nomura, Spread supersymmetry, J. High Energy Phys. 01 (2012) 082; L. J. Hall, Y. Nomura, and S. Shirai, Spread Supersymmetry with Wino LSP: Gluino and dark matter signals, ibid. 01 (2013) 036.

[36] A. Arvanitaki, N. Craig, S. Dimopoulos, and G. Villadoro, Mini-Split, J. High Energy Phys. 02 (2013) 126.

[37] M. R. Douglas, Basic results in vacuum statistics, C. R. Phys. 5, 965 (2004).

[38] V. Agrawal, S. M. Barr, J. F. Donoghue, and D. Seckel, The Anthropic principle and the mass scale of the standard model, Phys. Rev. D 57, 5480 (1998); Anthropic Considerations in Multiple Domain Theories and The Scale of Electroweak Symmetry Breaking, Phys. Rev. Lett. 80, 1822 (1998).

[39] H. Baer, V. Barger, M. Savoy, and H. Serce, The Higgs mass and natural supersymmetric spectrum from the landscape, Phys. Lett. B 758, 113 (2016).

[40] H. Baer, V. Barger, H. Serce, and K. Sinha, Higgs and superparticle mass predictions from the landscape, J. High Energy Phys. 03 (2018) 002.

[41] H. Baer, V. Barger, D. Sengupta, H. Serce, K. Sinha, and R. W. Deal, Is the magnitude of the Peccei-Quinn scale set by the landscape? arXiv:1905.00443 [hep-ph].

[42] K. R. Dienes, M. Lennek, D. Senechal, and V. Wasnik, Supersymmetry versus gauge symmetry on the Heterotic landscape, Phys. Rev. D 75, 126005 (2007); Is SUSY natural? New J. Phys. 10, 085003 (2008).

[43] L. Susskind, Supersymmetry breaking in the anthropic landscape in From Fields to Strings, edited by M. Shifman and J. Wheater (World Scientific, 2005), Vol. 3.

[44] F. Denef and M. R. Douglas, Distributions of flux vacua, J. High Energy Phys. 05 (2004) 072.

[45] K. J. Bae, H. Baer, V. Barger, and D. Sengupta, Revisiting the SUSY $\mu$ problem and its solutions in the LHC era, Phys. Rev. D 99, 115027 (2019).

[46] H. Baer, V. Barger, and D. Sengupta, Gravity safe, electroweak natural axionic solution to strong $C P$ and SUSY $\mu$ problems, Phys. Lett. B 790, 58 (2019).
[47] J. E. Kim and H. P. Nilles, The mu Problem and the Strong CP Problem, Phys. Lett. B 138, 150 (1984).

[48] H. M. Lee, S. Raby, M. Ratz, G. G. Ross, R. Schieren, K. Schmidt-Hoberg, and P. K. S. Vaudrevange, Discrete R symmetries for the MSSM and its singlet extensions, Nucl. Phys. B 850, 1 (2011).

[49] For a review, see, e.g., R. Arnowitt, and P. Nath, Developments in Supergravity Unified Models in Perspectives on Supersymmetry II, edited by G. L. Kane (World Scientific, 2010) and references therein; V. D. Barger, M. S. Berger, and P. Ohmann, Supersymmetric grand unified theories: Two loop evolution of gauge and Yukawa couplings, Phys. Rev. D 47, 1093 (1993); Supersymmetric particle spectrum, 49, 4908 (1994); G. L. Kane, C. F. Kolda, L. Roszkowski, and J. D. Wells, Study of constrained minimal supersymmetry, ibid. 49, 6173 (1994).

[50] F. E. Paige, S. D. Protopopescu, H. Baer, and X. Tata, ISAJET 7.69: A Monte Carlo event generator for pp, anti-p p, and e+ereactions, arXiv:hep-ph/0312045.

[51] K. L. Chan, U. Chattopadhyay, and P. Nath, Naturalness, weak scale supersymmetry and the prospect for the observation of supersymmetry at the Tevatron and at the CERN LHC, Phys. Rev. D 58, 096004 (1998).

[52] J. L. Feng, K. T. Matchev, and T. Moroi, Multi - TeV Scalars are Natural in Minimal Supergravity, Phys. Rev. Lett. 84, 2322 (2000); Focus points and naturalness in supersymmetry, Phys. Rev. D 61, 075005 (2000).

[53] D. Matalliotakis and H. P. Nilles, Implications of nonuniversality of soft terms in supersymmetric grand unified theories, Nucl. Phys. B 435, 115 (1995); M. Olechowski, and S. Pokorski, Electroweak symmetry breaking with nonuniversal scalar soft terms and large tan beta solutions, Phys. Lett. B 344, 201 (1995); P. Nath, and R. L. Arnowitt, Nonuniversal soft SUSY breaking and dark matter, Phys. Rev. D 56, 2820 (1997); J. R. Ellis, K. A. Olive, and Y. Santoso, The MSSM parameter space with nonuniversal Higgs masses, Phys. Lett. B 539, 107 (2002); J. R. Ellis, T. Falk, K. A. Olive, and Y. Santoso, Exploration of the MSSM with nonuniversal Higgs masses, Nucl. Phys. B 652, 259 (2003); H. Baer, A. Mustafayev, S. Profumo, A. Belyaev, and X. Tata, Direct, indirect and collider detection of neutralino dark matter in SUSY models with non-universal Higgs masses, J. High Energy Phys. 07 (2005) 065.

[54] W. Buchmuller, K. Hamaguchi,O. Lebedev, and M. Ratz, Supersymmetric Standard Model from the Heterotic String, Phys. Rev. Lett. 96, 121602 (2006); Supersymmetric standard model from the heterotic string (II), Nucl. Phys. B 785, 149 (2007); O. Lebedev, H. P. Nilles, S. Raby, S. Ramos-Sanchez, M. Ratz, P. K. S. Vaudrevange, and A. Wingerter, A Mini-landscape of exact MSSM spectra in heterotic orbifolds, Phys. Lett. B 645, 88 (2007); The Heterotic Road to the MSSM with R parity, Phys. Rev. D 77, 046013 (2008); O. Lebedev, H. P. Nilles, S. Ramos-Sanchez, M. Ratz, and P. K. S. Vaudrevange, Heterotic mini-landscape. (II). Completing the search for MSSM vacua in a Z(6) orbifold, Phys. Lett. B 668, 331 (2008); for a review, see H. P. Nilles, and P. K. S. Vaudrevange, Geography of fields in extra dimensions: String theory lessons for particle physics, Mod. Phys. Lett. A 30, 1530008 (2015).

[55] N. Arkani-Hamed, S. Dimopoulos, and S. Kachru, Predictive landscapes and new physics at a TeV, arXiv:hep-th/0501082.

[56] G. F. Giudice and R. Rattazzi, Living dangerously with lowenergy supersymmetry, Nucl. Phys. B 757, 19 (2006). 
[57] G. F. Giudice and A. Romanino, Split supersymmetry, Nucl. Phys. B 699, 65 (2004); Erratum to: "Split supersymmetry" [Nucl. Phys. B 699 (2004) 65] 706, 487 (2005).

[58] N. Arkani-Hamed, S. Dimopoulos, G. F. Giudice, and A. Romanino, Aspects of split supersymmetry, Nucl. Phys. B 709, 3 (2005).

[59] G. F. Giudice and A. Strumia, Probing high-scale and split supersymmetry with higgs mass measurements, Nucl. Phys. B 858, 63 (2012).

[60] A. Delgado and G. F. Giudice, On the tuning condition of split supersymmetry, Phys. Lett. B 627, 155 (2005).

[61] H. Baer, V. Barger, and P. Huang, Hidden SUSY at the LHC: The light higgsino-world scenario and the role of a lepton collider, J. High Energy Phys. 11 (2011) 031.

[62] Z. Han, G. D. Kribs, A. Martin, and A. Menon, Hunting quasidegenerate Higgsinos, Phys. Rev. D 89, 075007 (2014); H. Baer, A. Mustafayev, and X. Tata, Monojet plus soft dilepton signal from light higgsino pair production at LHC14, ibid. 90, 115007 (2014); C. Han, D. Kim, S. Munir, and M. Park, Accessing the core of naturalness, nearly degenerate higgsinos, at the LHC, J. High Energy Phys. 04 (2015) 132; H. Baer, V. Barger, M. Savoy, and X. Tata, Multichannel assault on natural supersymmetry at the high luminosity LHC, Phys. Rev. D 94, 035025 (2016).

[63] The ATLAS collaboration (ATLAS Collaboration), Searches for electroweak production of supersymmetric particles with compressed mass spectra in $\sqrt{s}=13 \mathrm{TeV} p p$ collisions with the ATLAS detector, ATLAS-CONF-2019-014.

[64] H. Baer, V. Barger, J. S. Gainer, P. Huang, M. Savoy, D. Sengupta, and X. Tata, Gluino reach and mass extraction at the LHC in radiatively-driven natural SUSY, Eur. Phys. J. C 77, 499 (2017).

[65] H. Baer, V. Barger, J. S. Gainer, H. Serce, and X. Tata, Reach of the high-energy LHC for gluinos and top squarks in
SUSY models with light Higgsinos, Phys. Rev. D 96, 115008 (2017).

[66] H. Baer, V. Barger, J. S. Gainer, D. Sengupta, H. Serce, and $\mathrm{X}$. Tata, LHC luminosity and energy upgrades confront natural supersymmetry models, Phys. Rev. D 98, 075010 (2018).

[67] H. Baer, V. Barger, P. Huang, D. Mickelson, A. Mustafayev, W. Sreethawong, and X. Tata, Same Sign Diboson Signature from Supersymmetry Models with Light Higgsinos at the LHC, Phys. Rev. Lett. 110, 151801 (2013).

[68] H. Baer, V. Barger, J. S. Gainer, M. Savoy, D. Sengupta, and X. Tata, Aspects of the same-sign diboson signature from wino pair production with light higgsinos at the high luminosity LHC, Phys. Rev. D 97, 035012 (2018).

[69] H. Baer, V. Barger, D. Mickelson, A. Mustafayev, and X. Tata, Physics at a higgsino factory, J. High Energy Phys. 06 (2014) 172; S. L. Lehtinen, H. Baer, M. Berggren, K. Fujii, J. List, T. Tanabe, and J. Yan, Naturalness and light Higgsinos: Why ILC is the right machine for SUSY discovery, arXiv:1710.02406 [hep-ph].

[70] R. D. Peccei and H. R. Quinn, CP Conservation in the Presence of Instantons, Phys. Rev. Lett. 38, 1440 (1977); R. D. Peccei, and H. R. Quinn, Constraints imposed by CP conservation in the presence of instantons, Phys. Rev. D 16, 1791 (1977); S. Weinberg, A New Light Boson? Phys. Rev. Lett. 40, 223 (1978); F. Wilczek, Problem of Strong $P$ and $T$ Invariance in the Presence of Instantons, ibid. 40, 279 (1978).

[71] K. J. Bae, H. Baer, and H. Serce, Prospects for axion detection in natural SUSY with mixed axion-higgsino dark matter: back to invisible? JCAP 06 (2017) 024.

[72] H. Baer, V. Barger, and D. Mickelson, Direct and indirect detection of higgsino-like WIMPs: concluding the story of electroweak naturalness, Phys. Lett. B 726, 330 (2013); H. Baer, V. Barger, and H. Serce, SUSY under siege from direct and indirect WIMP detection experiments, Phys. Rev. D 94, 115019 (2016). 